\title{
Keeping Avoider's graph almost acyclic
}

\author{
Dennis Clemens* Julia Ehrenmüller \\ Institut für Mathematik \\ Technische Universität Hamburg-Harburg \\ 21073 Hamburg, Germany \\ \{dennis.clemens, julia.ehrenmueller\}@tuhh.de \\ Yury Person \\ Institut für Mathematik \\ Tuan $\operatorname{Tran}^{\dagger}$ \\ Goethe-Universität \\ 60325 Frankfurt am Main, Germany \\ Institut für Mathematik \\ Freie Universität Berlin \\ person@math.uni-frankfurt.de \\ 14195 Berlin, Germany \\ tuan@math.fu-berlin.de
}

Submitted: Nov 28, 2014; Accepted: Feb 17, 2015; Published: Mar 6, 2015

Mathematics Subject Classification: 05C10, 05C57, 91A24

\begin{abstract}
We consider biased $(1: b)$ Avoider-Enforcer games in the monotone and strict versions. In particular, we show that Avoider can keep his graph being a forest for every but maybe the last round of the game if $b \geqslant 200 n \ln n$. By this we obtain essentially optimal upper bounds on the threshold biases for the non-planarity game, the non- $k$-colorability game, and the $K_{t}$-minor game thus addressing a question and improving the results of Hefetz, Krivelevich, Stojaković, and Szabó. Moreover, we give a slight improvement for the lower bound in the non-planarity game.
\end{abstract}

Keywords: positional games; Avoider-Enforcer; planarity game; threshold bias.

\section{Introduction}

Avoider-Enforcer games can be seen as the misère version of the well-known MakerBreaker games (studied first by Lehman [10], Chvátal and Erdős [6] and Beck [1, 3]). This means that, while playing according to their conventional rules, the players' goal is to lose the game. The general setting of Avoider-Enforcer games can be summarized as follows. Let $X$ be a finite set and let $\mathcal{F} \subseteq 2^{X}$. The two players, called Avoider and

*Supported by DFG project SZ 261/1-1.

†Supported by DFG within the Research Training Group "Methods for Discrete Structures". 
Enforcer, alternately occupy a certain number of elements of the so-called board $X$. The game ends when all elements are claimed by the players. Avoider wins if for every socalled losing set $F \in \mathcal{F}$, he does not occupy all elements of $F$ by the end of the game. Otherwise Enforcer wins. In particular, it is not possible that the game ends in a draw. We may assume that Avoider is always the first player since the choice of the player who is making the first move does not have an impact on our results.

In the following we will focus on games where the board $X$ is given by the edge set $E\left(K_{n}\right)$ of a complete graph and $\mathcal{F}_{n}$ is some graph property to be avoided. Following Hefetz, Krivelevich, Stojaković, and Szabó [8], we consider two different versions of Avoider-Enforcer games. Let $b$ be a positive integer. In the original, strict $(1: b)$ AvoiderEnforcer game (as investigated e.g. by Beck [2, 3], Hefetz, Krivelevich, and Szabó [9] and by $\mathrm{Lu}[11,12,13])$, Avoider occupies exactly 1 and Enforcer exactly $b$ unclaimed edges per round. If the number of unclaimed edges is strictly less than $b$ when it is Enforcer's turn, then he must select all the remaining unclaimed edges. For these strict rules, we define the lower threshold bias $f_{\mathcal{F}_{n}}^{-}$to be the largest integer such that Enforcer has a winning strategy for the $(1: b)$ game on $\left(E\left(K_{n}\right), \mathcal{F}_{n}\right)$ for every $b \leqslant f_{\mathcal{F}_{n}}^{-}$; and the upper threshold bias $f_{\mathcal{F}_{n}}^{+}$to be the smallest non-negative integer such that Avoider has a winning strategy for every $b>f_{\mathcal{F}_{n}}^{+}$. In general, $f_{\mathcal{F}_{n}}^{-}$and $f_{\mathcal{F}_{n}}^{+}$do not coincide as shown by Hefetz, Krivelevich, and Szabó [9].

In the monotone $(1: b)$ Avoider-Enforcer game, Avoider occupies at least 1 and Enforcer at least $b$ unclaimed edges per round. Again, if the number of unclaimed edges is strictly less than $b$ when it is Enforcer's turn, then he must select all the remaining unclaimed edges. Games with these monotone rules are bias monotone, as it was shown by Hefetz, Krivelevich, Stojaković, and Szabó in [8]. This means that there exists a unique threshold bias $f_{\mathcal{F}_{n}}^{m o n}$ which is defined as the non-negative integer for which Enforcer wins the monotone $(1: b)$ game if and only if $b \leqslant f_{\mathcal{F}_{n}}^{m o n}$.

One might wonder at this point whether for any family $\mathcal{F}_{n}$ there is some general relation between the three thresholds mentioned above like $f_{\mathcal{F}_{n}}^{-} \leqslant f_{\mathcal{F}_{n}}^{\text {mon }} \leqslant f_{\mathcal{F}_{n}}^{+}$. Indeed, if $\mathcal{F}_{n}=\mathcal{F}_{P_{3}, n}$ is the family of all paths on 3 vertices of $K_{n}$, then these inequalities hold, as shown by Hefetz, Krivelevich, Stojaković, and Szabó in [8]. However, these inequalities are not true in general and in fact the outcome of some Avoider-Enforcer games in the strict setting can differ a lot from the outcome of the corresponding monotone games. For instance, it was also shown in [8] and by Hefetz, Krivelevich, and Szabó in [9] that for the Avoider-Enforcer connectivity game, where $\mathcal{F}_{n}=C_{n}$ is the family of all spanning trees of $K_{n}$, we have $f_{C_{n}}^{\text {mon }}=(1+o(1)) \frac{n}{\ln n}$, while $f_{C_{n}}^{+}=f_{C_{n}}^{-}=\left\lfloor\frac{n-1}{2}\right\rfloor$.

In the present paper, we will be studying biased strict and monotone Avoider-Enforcer games, where Avoider's goal is to maintain an (almost) acyclic graph. This will have a series of improvements on the bias of various games such as planarity, colorability and minor games. Before stating our results we survey the relevant developments so far.

Define $N C_{n}^{k}$ to be the set consisting of the edge sets of all non- $k$-colorable graphs on $n$ vertices. It was proved by Hefetz, Krivelevich, Stojaković, and Szabó [7] that for every 
$k \geqslant 3$, Avoider can win the strict $(1: b)$ "non- $k$-colorability" game $N C_{n}^{k}$ against any bias larger than $2 k n^{1+\frac{1}{2 k-3}}$. On the other hand, it was shown by the same authors [7] that there exists a constant $s_{k}$ such that Enforcer has a strategy to win the game for every $b \leqslant s_{k} n$. Moreover, in the same paper the authors mention that there exists a constant $c>0$ such that $c n \leqslant f_{N C_{n}^{2}}^{-} \leqslant f_{N C_{n}^{2}}^{+} \leqslant n^{3 / 2}$.

Let $M_{n}^{t}$ denote the set of all edge sets of all graphs on $n$ vertices containing a $K_{t}$-minor. Playing against a bias larger than $2 n^{5 / 4}$, Avoider can win the strict $(1: b) K_{t}$-minor game $M_{n}^{t}$ for every $t \geqslant 4$ whereas if $b$ is almost as large as $n / 2$ Enforcer has a winning strategy where $t$ is some constant power of $n$, see Hefetz et al. [7]. It was proved by Hefetz, Krivelevich, Stojaković, and Szabó in [8] that the threshold bias for the monotone version is of order $n^{3 / 2}$ for $t=3$.

Finally, let us introduce the "non-planarity" Avoider-Enforcer game. Let $N P_{n}$ be the set consisting of the edge sets of all non-planar graphs on $n$ vertices. In the so-called "non-planarity" game $N P_{n}$, Avoider's task is to keep his graph planar. Hefetz et al. proved in [7] that in the strict $(1: b)$ non-planarity game, Avoider can succeed against any bias larger than $2 n^{5 / 4}$. Furthermore, their proof also can be applied when considering the monotone rules instead.

The main results of our paper are the following two theorems. The first theorem gives a lower bound of $200 n \ln n$ on the bias such that both in the monotone and in the strict $(1: b)$ Avoider-Enforcer game, Avoider can keep his graph acyclic apart from at most one unicyclic component.

Theorem 1. For $n$ sufficiently large and $b \geqslant 200 n \ln n$, Avoider can ensure that both in the monotone and in the strict $(1: b)$ Avoider-Enforcer game by the end of the game Avoider's graph is a forest plus at most one additional edge.

In the strict $(1: b)$ game stated in the theorem below, Avoider's task is to keep his graph acyclic for which he has again a winning strategy for some bias $b$ between $200 n \ln n$ and $201 n \ln n$.

Theorem 2. For $n$ sufficiently large, there is a bias $200 n \ln n \leqslant b \leqslant 201 n \ln n$ such that Avoider can ensure that in the strict (1:b) Avoider-Enforcer game by the end of the game Avoider's graph is a forest.

While these results are interesting in their own right, they can be applied directly to three other games discussed above: the "non- $k$-colorability", the " $K_{t}$-minor", and the "non-planarity" Avoider-Enforcer games.

The two corollaries below are direct consequences of our main theorems above. In particular, these results improve upper bounds for $f_{N C_{n}^{k}}^{+}$and $f_{N C_{n}^{k}}^{m o n}$ with $k \geqslant 3$, and for $f_{N C_{n}^{2}}^{-}$. Furthermore better bounds are obtained for $f_{M_{n}^{t}}^{+}$and $f_{M_{n}^{t}}^{m o n}$ with $t \geqslant 4$ and for $f_{M_{n}^{3}}^{-}$. Finally, the bounds on $f_{N P_{n}}^{+}$and $f_{N P_{n}}^{m o n}$ are improved as well. 
Corollary 3. For $n$ sufficiently large and $b \geqslant 200 n \ln n$, Avoider can ensure that in the monotone/strict (1:b) Avoider-Enforcer game by the end of the game his graph is planar, $k$-colorable for $k \geqslant 3$, and does not contain a $K_{t}$-minor for $t \geqslant 4$. Thus,

$$
f_{N P_{n}}^{+}, f_{N C_{n}^{k}}^{+}, f_{M_{n}^{t}}^{+}, f_{N P_{n}}^{m o n}, f_{N C_{n}^{k}}^{m o n}, f_{M_{n}^{t}}^{m o n} \leqslant 200 n \ln n .
$$

Proof. By Theorem 1, Avoider can ensure that by the end of the game his graph is a forest plus at most one additional edge. Clearly, this graph is planar, 3-colorable, and does not contain a $K_{4}$-minor, proving the statement.

Corollary 4. For $n$ sufficiently large, there is a bias $200 n \ln n \leqslant b \leqslant 201 n \ln n$ such that Avoider can ensure that in the strict $(1: b)$ Avoider-Enforcer game by the end of the game Avoider's graph is 2-colorable and does not contain a $K_{3}$-minor. Thus,

$$
f_{N C_{n}^{2}}^{-}, f_{M_{n}^{3}}^{-}=O(n \ln n)
$$

Proof. By Theorem 2, Avoider can ensure that by the end of the game his graph is a forest. Obviously, this graph is 2-colorable and does not contain a $K_{3}$-minor, proving the statement.

Hefetz, Krivelevich, Stojaković, and Szabó conjectured in [7] that the Avoider-Enforcer non-planarity, non- $k$-colorability and the $K_{t}$-minor games should be asymptotically monotone as $n$ tends to infinity. That is their upper and lower threshold should be of the same order, i.e. $f_{\mathcal{F}_{n}}^{-}=\Theta\left(f_{\mathcal{F}_{n}}^{+}\right)$. Since in each of the three games we have lower bounds on $f_{\mathcal{F}_{n}}^{-}$ that are linear in $n$, Corollary 3 and Corollary 4 show that the threshold biases are at most $O(\ln n)$ factor apart, thus giving additional evidence that this conjecture might be true.

Coming back to the $(1: b)$ non-planarity Avoider-Enforcer game, it was also proved in [7] that in the strict version Enforcer can win whenever $b \leqslant \frac{n}{2}-o(n)$. Moreover, with a slight modification of the proof, the same result can be obtained for the monotone rules. We improve this bound as well.

Proposition 5. For $n$ sufficiently large and $b \leqslant 0.59 n$, Enforcer can ensure that both in the monotone and in the strict $(1: b)$ Avoider-Enforcer game, Avoider creates a nonplanar graph. Thus,

$$
0.59 n \leqslant f_{N P_{n}}^{m o n}, f_{N P_{n}}^{-}
$$

It should be mentioned that for the sake of readability, we do not optimize the constants in our theorems and proofs. Our graph-theoretic notation is standard and follows [5]. In particular, given a graph $G$ its vertex set is denoted by $V(G)$ and its edge set by $E(G)$. The rest of the paper is organized as follows. In Section 2 we prove the two main results, namely Theorem 1 and Theorem 2. In Section 3 we study the non-planarity Avoider-Enforcer game and prove Proposition 5. Finally, in Section 4 we discuss some open problems. 


\section{Forests and almost forests}

Proof of Theorem 1. Let $n$ be large enough and let $b \geqslant 200 n \ln n$. In the following we will provide Avoider with a strategy that ensures that by the end of the game Avoider's graph is a forest plus at most one additional edge.

Let $t$ be the smallest integer with

$$
n\left(\frac{t+1}{10 \ln n}\right)^{t}<3 .
$$

An easy calculation shows that $t=\Theta(\ln n)$, in particular, we have for large $n$ that

$$
t<\ln n / 3
$$

To succeed, Avoider will play according to $t$ stages in increasing order and each stage consists of several consecutive rounds where it is possible that a stage lasts zero rounds, i.e. that a stage does not occur at all. In the first $t-1$ stages, Avoider always claims exactly one edge in each round, connecting two components of his forest such that the sum of their sizes is minimal (whenever we talk about components, we mean the components of Avoider's forest). In the last stage, which will be shown to last at most one round, Avoider will claim an arbitrary further edge. We refer to edges, neither taken by Avoider nor by Enforcer, as unclaimed edges.

Starting with Stage 1, Avoider plays according to the following rules.

Stage $\boldsymbol{k}$ (for $\boldsymbol{k} \in[\boldsymbol{t}-\mathbf{1}]$ ). If there exists an unclaimed edge $e$ between two components $T_{1}$ and $T_{2}$ with $\left|V\left(T_{1}\right)\right|+\left|V\left(T_{2}\right)\right|=k+1$, Avoider claims such an edge, thus creating a component on the vertex set $V\left(T_{1}\right) \cup V\left(T_{2}\right)$. Then it is Enforcer's turn and the round is over.

Avoider is going to play according to Stage $k$ in the next round as well. If there is no such edge $e$ to be claimed at Stage $k$, Avoider proceeds with Stage $k+1$. (As mentioned above it might happen that there is no edge to be claimed at Stage $k$ already when Avoider enters Stage $k$. In that case, this stage lasts zero rounds, and Avoider immediately proceeds with Stage $k+1$.)

Stage $\boldsymbol{t}$. In every further round, Avoider claims exactly one arbitrary free edge.

One can easily verify that Avoider can follow the strategy. Moreover, as long as Avoider plays according to the strategy of the first $t-1$ stages, his graph remains a forest. Thus, in order to show that the above described strategy is indeed a winning strategy, it remains to show that the last stage lasts at most one round. As a first step we aim to bound the number of rounds a given stage lasts. Let $n_{k}$ denote the number of rounds in Stage $k-1$. Observe that Avoider creates components of size exactly $k$ only in this stage. Thus, the number of such components is always bounded from above by $n_{k}$.

Claim 6. For every $k \leqslant t$,

$$
n_{k} \leqslant n\left(\frac{k}{10 \ln n}\right)^{k-1}
$$


Proof. The claim is obviously true for $k=1$. So, let $k>1$ and we proceed by induction. When Avoider enters Stage $k-1$ every existing component contains at most $k-1$ vertices and there are no unclaimed edges between any two components $T_{1}$ and $T_{2}$ with $\left|V\left(T_{1}\right)\right|+$ $\left|V\left(T_{2}\right)\right| \leqslant k-1$. In particular, every unclaimed edge is either between two components $T_{1}$ and $T_{2}$ with $\left|V\left(T_{1}\right)\right|+\left|V\left(T_{2}\right)\right| \geqslant k$ or between two vertices within the same component which has size at most $k-1$. The first case contributes at most $\sum_{1 \leqslant i \leqslant j \leqslant k-1: i+j \geqslant k} i j n_{i} n_{j}$ unclaimed edges since $n_{i}$ is an upper bound on the number of components of size exactly $i$. For the second case we find an upper bound of $(k-1) n$ by the following reason: Let $n_{i}^{\prime}$ denote the number of components of order $i$ immediately after the end of Stage $k-1$. Then the number of unclaimed edges within components after $k-1$ stages is at most $\sum_{i=1}^{k}\left(\begin{array}{l}i \\ 2\end{array}\right) n_{i}^{\prime} \leqslant(k-1) \sum_{i=1}^{k} i n_{i}^{\prime}=(k-1) n$, since $\sum_{i=1}^{k} i n_{i}^{\prime}=n$.

Therefore, at the beginning of Stage $k-1$, the number of unclaimed edges is at most $\sum_{1 \leqslant i \leqslant j \leqslant k-1: i+j \geqslant k} i j n_{i} n_{j}+(k-1) n$. Since in each but possibly the last round at least $b+1$ edges are claimed ( 1 by Avoider and $b$ by Enforcer), we conclude

$$
n_{k} \leqslant \frac{1}{b+1}\left(\sum_{1 \leqslant i \leqslant j \leqslant k-1: i+j \geqslant k} i j n_{i} n_{j}+(k-1) n\right)+1 .
$$

We use the induction hypothesis to estimate the sum $\sum_{1 \leqslant i \leqslant j \leqslant k-1: i+j=s} i j n_{i} n_{j}$ for $s=$ $k, \ldots, 2 k-2$ as follows:

$$
\sum_{\substack{1 \leqslant i \leqslant j \leqslant k-1 \\ i+j=s}} i j n_{i} n_{j} \leqslant \frac{n^{2}}{(10 \ln n)^{s-2}} \sum_{\substack{1 \leqslant i \leqslant j \leqslant k-1 \\ i+j=s}} i^{i} j^{j} \leqslant \frac{n^{2}}{(10 \ln n)^{s-2}} \sum_{\substack{1 \leqslant i \leqslant j \leqslant s-1 \\ i+j=s}} i^{i} j^{j} .
$$

For $s \leqslant 6$, it is easy to check that

$$
\sum_{\substack{1 \leqslant i \leqslant j \leqslant s-1 \\ i+j=s}} i^{i} j^{j}<3 s^{s-1} .
$$

On the other hand, for $s \geqslant 7$ observe that we have for every $2 \leqslant i \leqslant s / 2$

$$
\left(\frac{i}{s}\right)^{i} \leqslant\left(\frac{2}{s}\right)^{2}
$$

by an easy calculation for $i \leqslant 3$ and since

$$
\frac{i^{i}}{s^{i-2}} \leqslant \frac{i^{i}}{(2 i)^{i-2}} \leqslant \frac{i^{2}}{2^{i-2}} \leqslant 4
$$

for every $i \geqslant 4$. Therefore, we also obtain for $s \geqslant 7$

$$
\begin{aligned}
\sum_{\substack{1 \leqslant i \leqslant j \leqslant s-1 \\
i+j=s}} i^{i} j^{j}<s^{s-1}+\sum_{2 \leqslant i \leqslant s / 2} i^{i} s^{s-i} & =s^{s-1}\left(1+s \sum_{2 \leqslant i \leqslant s / 2}\left(\frac{i}{s}\right)^{i}\right) \\
& \stackrel{(6)}{\leqslant} s^{s-1}\left(1+s \sum_{2 \leqslant i \leqslant s / 2}\left(\frac{2}{s}\right)^{2}\right)<3 s^{s-1} .
\end{aligned}
$$


Observing that

$$
\begin{aligned}
\left(\frac{s}{10 \ln n}\right)^{s-1} & =\left(\frac{k}{10 \ln n}\right)^{k-1} \prod_{i=1}^{s-k} \frac{k+i-1}{10 \ln n}\left(1+\frac{1}{k+i-1}\right)^{k+i-1} \\
& \leqslant\left(\frac{k}{10 \ln n}\right)^{k-1}\left(\frac{2 k e}{10 \ln n}\right)^{s-k} \stackrel{(2)}{\leqslant}\left(\frac{k}{10 \ln n}\right)^{k-1} 2^{k-s}
\end{aligned}
$$

we can simplify (3) using $b \geqslant 200 n \ln n$ and Equations (4), (5), and (7) as follows:

$$
\begin{aligned}
n_{k} & \leqslant \frac{1}{200 n \ln n}\left(\sum_{s=k}^{2 k-2} 30 n^{2} \ln n\left(\frac{s}{10 \ln n}\right)^{s-1}+(k-1) n\right)+1 \\
& \stackrel{(8)}{\leqslant} \frac{3 n}{20}\left(\frac{k}{10 \ln n}\right)^{k-1} \sum_{s=k}^{2 k-2} 2^{k-s}+\frac{k-1}{200 \ln n}+1 \\
& \stackrel{(2)}{\leqslant} \frac{3 n}{10}\left(\frac{k}{10 \ln n}\right)^{k-1}+2 \stackrel{(1)}{\leqslant} n\left(\frac{k}{10 \ln n}\right)^{k-1} .
\end{aligned}
$$

This completes the proof of Claim 6 .

Now, analogously to the calculation of the proof of Claim 6 it follows that, when Avoider enters the last stage, Stage $t$, the number of remaining unclaimed edges is bounded by

$$
\begin{aligned}
\sum_{\substack{1 \leqslant i \leqslant j \leqslant t \\
i+j \geqslant t+1}} i j n_{i} n_{j}+t n & \leqslant \sum_{s=t+1}^{2 t} 30 n^{2} \ln n\left(\frac{t+1}{10 \ln n}\right)^{t} 2^{t+1-s}+t n \\
& \stackrel{(1)}{\leqslant} 180 n \ln n+t n<200 n \ln n
\end{aligned}
$$

by the choice of $t(t<\ln n / 3)$ and for $n$ sufficiently large. Thus, this last stage lasts at most one round.

Now we turn to the case of the strict rules, when Enforcer has to claim exactly $b$ edges during each round (except possibly for the last one).

Proof of Theorem 2. We will show below that for large enough $n$, there exists $b$ with $200 n \ln n \leqslant b \leqslant 201 n \ln n$ and the remainder of $\left(\begin{array}{l}n \\ 2\end{array}\right)$ divided by $b+1$ is at least $n \ln n$.

Before proving this claim let us explain how the theorem follows then. Let $b$ be given as above. Avoider now plays according to the same strategy as given in the proof of Theorem 1 until he reaches Stage $t$, where again $t$ is the smallest integer with $n\left(\frac{t+1}{10 \ln n}\right)^{t}<3$. At this point, Avoider's graph is still a forest, the components of which are all of size at most $t$. Analogously to the proof of Theorem1, there can be at most $t n<n \ln n / 3$ unclaimed edges within components. However, since the remainder of the division $\left(\begin{array}{l}n \\ 2\end{array}\right) /(b+1)$ is at least $n \ln n$, there exist unclaimed edges connecting two different components when 
Avoider enters Stage $t$ (provided $n$ is large enough). Now, Avoider just claims one such edge arbitrarily. His graph remains a forest and afterwards, Enforcer must take all remaining edges. Observe that in the case when Avoider is the second player, he does not even claim an edge in the last round.

So, it only remains to prove the above mentioned claim. Let $b_{1}=\lceil 200.5 n \ln n\rceil$. Moreover, let

$$
\left(\begin{array}{l}
n \\
2
\end{array}\right)=q_{1}\left(b_{1}+1\right)+r_{1} \text { with } 0 \leqslant r_{1} \leqslant b_{1} \text { and } q_{1} \sim \frac{n}{401 \ln n} .
$$

If $r_{1}>n \ln n$, we are done by setting $b=b_{1}$. Otherwise, let $b=b_{1}-\left\lceil 402 \ln ^{2} n\right\rceil$. Then

$$
\left(\begin{array}{l}
n \\
2
\end{array}\right)=q_{1}(b+1)+\left(r_{1}+q_{1}\left\lceil 402 \ln ^{2} n\right\rceil\right) .
$$

Moreover, for large enough $n$, we obtain $r_{1}+q_{1}\left\lceil 402 \ln ^{2} n\right\rceil<b$, and therefore the remainder of the division $\left(\begin{array}{l}n \\ 2\end{array}\right)$ by $(b+1)$ is at least $r_{1}+q_{1}\left\lceil 402 \ln ^{2} n\right\rceil>n \ln n$, while $200 n \ln n \leqslant b \leqslant$ $201 n \ln n$.

\section{Lower bound in the non-planarity game}

Before obtaining a lower bound for the non-planarity Avoider-Enforcer game in Proposition 5, we analyze another strict game where two players, the first player (denoted by $\mathrm{FP}$ ) and the second player (denoted by SP), claim exactly 1 and $b$ edges, respectively.

Proposition 7. Let $c=1 / 1000$. For $n$ sufficiently large and every $0.49 n \leqslant b \leqslant 0.59 n$ the second player in a strict $(1: b)$ game on $E\left(K_{n}\right)$ can isolate at least

$$
n-(1-c) \frac{n^{2}}{2 b} \quad \text { vertices }
$$

i.e. claim all edges that are incident to these vertices.

Proof. Case 1. (0.49n $\leqslant \mathbf{b} \leqslant \mathbf{0 . 5 5 n}$.) As long as there are at least 4 vertices not isolated by the second player (SP) and not touched by the first player (FP), SP can isolate a vertex in every fourth round. Indeed, assume SP isolated a vertex in the previous round and now wants to isolate one vertex within the next 4 rounds. He fixes 4 vertices $v_{1}, v_{2}, v_{3}, v_{4}$ that are neither isolated by him nor touched by FP. In every first round, SP claims all edges between these 4 vertices and at each $v_{i}$ he additionally claims $\lfloor(b-6) / 4\rfloor$ arbitrary incident edges. Now, it is FP's turn. He can touch at most one of these four vertices since all edges between them are already claimed by SP. Without loss of generality, $v_{1}, v_{2}$, and $v_{3}$ are still untouched by FP. Now in the second round SP claims at each of these three vertices $\lfloor b / 3\rfloor$ arbitrary incident edges. Again, FP can touch at most one of these three vertices in his turn. Without loss of generality, $v_{1}$ and $v_{2}$ are still untouched by FP after that. In the third round, SP claims at each of these two vertices $\lfloor b / 2\rfloor$ arbitrary incident edges. After 
FP's next turn, w. l. o.g. $v_{1}$ is still untouched by FP. Now, SP simply claims all remaining incident edges at $v_{1}$, which is possible since $3+\lfloor(b-6) / 4\rfloor+\lfloor b / 3\rfloor+\lfloor b / 2\rfloor+b>n$, for large $n$. Note that while SP isolates one vertex, FP can touch at most 8 other vertices. It follows that the number of vertices that SP isolates in total is at least $\lfloor n / 9\rfloor \geqslant n-(1-c) \frac{n^{2}}{2 b}$.

Case 2. $(\mathbf{0 . 5 5 n} \leqslant \mathbf{b} \leqslant \mathbf{0 . 5 8 n}$.) Analogously to Case 1 , SP can isolate a vertex in every third round as long as there are at least 3 vertices not touched by FP. This time, $\mathrm{SP}$ starts by only fixing three vertices $v_{1}, v_{2}, v_{3}$ and isolates then one of them within three rounds, which is possible since $2+\lfloor(b-3) / 3\rfloor+\lfloor b / 2\rfloor+b>n$, for large $n$. It follows then that SP isolates at least $\lfloor n / 7\rfloor \geqslant n-(1-c) \frac{n^{2}}{2 b}$ vertices in total.

Case 3. $(\mathbf{0 . 5 8 n} \leqslant \mathbf{b} \leqslant \mathbf{0 . 5 9 n}$.) Analogously to Case 2, SP can isolate a vertex in every third round as long as there are at least 3 vertices not touched by FP. In a first phase, SP follows the above described strategy and he isolates $n-1.5 b$ vertices, which happens in at most $3 n-4.5 b$ rounds. During this phase, FP can touch at most $6 n-9 b$ vertices. Afterwards, for every vertex that is neither isolated by SP nor touched by FP, $\mathrm{SP}$ only needs to claim at most $1.5 b$ further incident edges in order to isolate it. But then, analogously to the previous cases, SP can isolate one vertex in every second round, since $1+\lfloor(b-1) / 2\rfloor+b \geqslant 1.5 b$. Thus, in the second phase after at most $3 n-4.5 b$ rounds, SP isolates a vertex in every second round as long as possible. Since at the beginning of the second phase at least $n-(n-1.5 b)-(6 n-9 b)=10.5 b-6 n$ vertices were neither isolated by SP nor touched by FP, SP can isolate at least $(10.5 b-6 n) / 5$ further vertices. In total $\mathrm{SP}$ will isolate at least $(n-1.5 b)+(10.5 b-6 n) / 5 \geqslant n-(1-c) \frac{n^{2}}{2 b}$ vertices.

Lemma 8. For $n$ sufficiently large and $b \leqslant 0.59 n$ Enforcer can ensure that in the strict $(1: b)$ game on $E\left(K_{n}\right)$ Avoider creates a non-planar graph. Thus,

$$
0.59 n \leqslant f_{N P_{n}}^{-}
$$

Proof. Since the statement is already proved for $b \leqslant 0.49 n$ in [7], we may assume from now on that $0.49 n \leqslant b$. The following proof will be a slight modification of the one given in [7]. Let $c=1 / 1000$ be as in Proposition 7 and choose an integer $k \geqslant 3$ such that

$$
\frac{k}{k-2}\left(1-\frac{c}{2}\right)<1 \text {. }
$$

Enforcer's strategy consists of two goals: First of all, he wants to prevent Avoider from creating cycles of length at most $k$. Secondly, he wants to isolate a large number of vertices to ensure that Avoider's graph lives on a small vertex set. For this he splits his bias $b=b_{1}+b_{2}$ ( $b_{1}$ and $b_{2}$ will be chosen later) and uses $b_{1}$ for his first goal, and $b_{2}$ for the second goal.

Preventing cycles. It follows from the work of Bednarska and Luczak [4] (see also the proof of Theorem 2.3 in [7]), that for every $3 \leqslant i \leqslant k$ there is a constant $c_{i}$ such that, for sufficiently large $n$, Enforcer can prevent Avoider from claiming a cycle of length $i$ if Enforcer is allowed to claim at least $c_{i} n^{\frac{i-2}{i-1}}$ edges. Let $C=\max \left\{c_{i}: 3 \leqslant i \leqslant k\right\}$. Then, simultaneously playing according to the different strategies for preventing cycles of length 
$3 \leqslant i \leqslant k$, Enforcer can ensure that Avoider's graph has girth larger than $k$ if he claims at least

$$
\sum_{i=3}^{k} c_{i} n^{\frac{i-2}{i-1}} \leqslant C k n^{\frac{k-2}{k-1}}=: b_{1}
$$

edges per round. Observe that $b_{1}=o(b)$.

Isolating vertices. Let $b_{2}=b-b_{1}=b(1-o(1))$. In each round Enforcer uses $b_{2}$ edges to play according to the strategy given in the proof of Proposition 7 . Therefore, he isolates at least $n-(1-c) \frac{n^{2}}{2 b_{2}} \geqslant n-\left(1-\frac{c}{2}\right) \frac{n^{2}}{2 b}$ vertices.

Now, let Enforcer split his bias $b=b_{1}+b_{2}$, and thus play so as to prevent cycles of length at most $k$, while at the same time to isolate at least $n-\left(1-\frac{c}{2}\right) \frac{n^{2}}{2 b}$ vertices. Notice that it does not hurt Enforcer if the combination of the above strategies leads to claiming the same edge more than once - Enforcer can claim an arbitrary edge instead since this does not destroy the properties of the graph he is about to create. Let $A$ be Avoider's graph at the end of the game. We know that $|V(A)| \leqslant\left(1-\frac{c}{2}\right) \frac{n^{2}}{2 b}$ and $\operatorname{girth}(A)>k$. If $A$ was planar, then, by a standard application of Euler's formula, we would have

$$
|E(A)|<\frac{k}{k-2}(|V(A)|-2)<\frac{k}{k-2}\left(1-\frac{c}{2}\right) \frac{n^{2}}{2 b} .
$$

However, by the number of rounds the game lasts, we have

$$
|E(A)| \geqslant\left\lfloor\frac{\left(\begin{array}{l}
n \\
2
\end{array}\right)}{b+1}\right\rfloor>\frac{k}{k-2}\left(1-\frac{c}{2}\right) \frac{n^{2}}{2 b},
$$

using (9), for $n$ sufficiently large. Thus, Avoider's graph is non-planar and Enforcer wins.

Lemma 9. For $n$ sufficiently large and $b \leqslant 0.59 n$ Enforcer can ensure that Avoider creates a non-planar graph in the monotone $(1: b)$ game on $E\left(K_{n}\right)$. Thus,

$$
0.59 n \leqslant f_{N P_{n}}^{m o n} .
$$

Proof. Let $A$ be Avoider's graph throughout the game, and let $A^{*} \subseteq A$ be a subgraph consisting of exactly one edge from every round played so far. Enforcer claims in every round exactly $b^{\prime}:=\max \{0.49 n, b\}$ edges according to the strategy given in the proof of the previous lemma, assuming $A^{*}$ to be Avoider's graph. If this strategy asks Enforcer to claim an edge from $A \backslash A^{*}$, he will claim another arbitrary edge instead. We distinguish two cases.

Case 1. $|\mathbf{E}(\mathbf{A})|>$ 3n. Then, by Euler's formula, Avoider's graph is non-planar and Enforcer wins.

Case 2. $|\mathbf{E}(\mathbf{A})| \leqslant \mathbf{3 n}$. Then the number of rounds the game lasts is at least $\frac{\left(\begin{array}{l}n \\ 2\end{array}\right)-3 n}{b^{\prime}}=$ $\frac{n^{2}}{2 b^{\prime}}(1-o(1))$, which also gives

$$
\left|E\left(A^{*}\right)\right| \geqslant \frac{n^{2}}{2 b^{\prime}}(1-o(1)) .
$$


By the above described strategy we get again, similar to the proof of Lemma $8,\left|V\left(A^{*}\right)\right| \leqslant$ $\left(1-\frac{c}{2}\right) \frac{n^{2}}{2 b^{\prime}}$ as well as $\operatorname{girth}\left(A^{*}\right)>k$, ensuring that $A^{*}$ cannot be planar provided that $n$ is large enough.

Proof of Proposition 5. This proposition follows directly from Lemma 8 and Lemma 9.

\section{Open questions}

For each of the games considered for Corollary 3, we have shown that the lower and upper threshold bias differ at most by a factor of $\ln n$. However, we believe that this factor can be replaced by some constant. We wonder whether this can already be done for the strategy we analyzed in the proof of Theorem 1, where we have shown that Avoider can keep his graph almost acyclic.

Question 10. Is there a constant $C>0$ such that the following holds: For $n$ sufficiently large and $b \geqslant C n$, Avoider has a strategy that creates at most one cycle in the monotone/strict $(1: b)$ game?

In case the question above can be answered positively, the following conjecture would follow immediately.

Conjecture 11 ([7]). The Avoider-Enforcer non-planarity, non-k-colorability and $K_{t^{-}}$ minor games are asymptotically monotone for every $k \geqslant 3$ and $t \geqslant 4$.

Our result on the lower threshold bias for the non-planarity game is obtained by splitting Enforcer's strategy into two parts. The first part, based on the strategy from [7], is to prevent small cycles in Avoider's graph. The second part is to isolate a large number of vertices. So, our improvement was obtained by studying a positional game in which one player has the goal to isolate as many vertices as possible. This game itself seems to be of interest.

Question 12. Let $b \in \mathbb{N}$. What is the largest number of vertices that the second player can isolate in a $(1: b)$ game on $E\left(K_{n}\right)$ under the strict rules?

\section{Acknowledgement}

We are grateful to Miloš Stojaković for helping us to further improve our bounds in Theorem 1 and Theorem 2.

\section{References}

[1] J. Beck. Remarks on positional games. I. Acta Math. Acad. Sci. Hungar., 40(1-2):6571, 1982. 
[2] J. Beck. Ramsey games. Discrete Math., 249(1-3):3-30, 2002, Combinatorics, graph theory and computing (Louisville, KY, 1999).

[3] J. Beck. Combinatorial games. Encyclopedia of Mathematics and its Applications, vol. 114, Cambridge University Press, Cambridge, 2008, Tic-tac-toe theory.

[4] M. Bednarska and T. Euczak. Biased positional games for which random strategies are nearly optimal. Combinatorica 20 (4):477-488, 2000.

[5] B. Bollobás. Modern graph theory. Graduate Texts in Mathematics, vol. 184, Springer-Verlag, New York, 1998.

[6] V. Chvátal and P. Erdős. Biased positional games. Ann. Discrete Math., 2:221-229, 1978, Algorithmic aspects of combinatorics (Conf., Vancouver Island, B.C., 1976).

[7] D. Hefetz, M. Krivelevich, M. Stojaković, and T. Szabó. Planarity, colorability, and minor games. SIAM J. Discrete Math., 22(1):194-212, 2008.

[8] D. Hefetz, M. Krivelevich, M. Stojaković, and T. Szabó. Avoider-Enforcer: the rules of the game. J. Combin. Theory Ser. A, 117(2): 152-163, 2010.

[9] D. Hefetz, M. Krivelevich, and T. Szabó. Avoider-Enforcer games. J. Combin. Theory Ser. A, 114(5):840-853, 2007.

[10] A. Lehman. A solution of the Shannon switching game. J. Soc. Indust. Appl. Math., 12:687-725, 1964.

[11] X. Lu. A matching game. Discrete Math., 94(3):199-207, 1991.

[12] X. Lu. Hamiltonian games. J. Combin. Theory Ser. B, 55(1):18-32, 1992.

[13] X. Lu. A Hamiltonian game on $K_{n, n}$. Discrete Math. 142(1-3):185-191, 1995. 